\title{
Tracking Late Jurassic ornithopods in the Lusitanian Basin of Portugal: Ichnotaxonomic implications
}

\author{
Diego Castanera, Bruno C. Silva, Vanda F. Santos, Elisabete Malafaia, and Matteo Belvedere \\ Acta Palaeontologica Polonica 65 (2), 2020: 399-412 doi:https://doi.org/10.4202/app.00707.2019
}

The Sociedade de História Natural in Torres Vedras, Portugal houses an extensive collection of as yet undescribed dinosaur tracks with ornithopod affinities. They have been collected from different Late Jurassic (Kimmeridgian-Tithonian) geological formations (Praia de Amoreira-Porto Novo, Alcobaça, Sobral, and Freixial) that outcrop along the Portuguese coast, and belong to two different sub-basins of the Lusitanian Basin (the Consolação and Turcifal sub-basins). Three main morphotypes can be distinguished on the basis of size, mesaxony and the morphology of the metatarsophalangeal pad impression. The minute to small-sized morphotype is similar to the Anomoepus-like tracks identified in other Late Jurassic areas. The small to medium-sized morphotype resembles the Late Jurassic-Early Cretaceous ichnotaxon Dinehichnus, already known in the Lusitanian Basin. Interestingly, these two morphotypes can be distinguished qualitatively (slightly different size, metatarsophalangeal pad impression and digit morphology) but are nevertheless difficult to discriminate by quantitatively analysing their length-width ratio and mesaxony. The third morphotype is considered a large ornithopod footprint belonging to the ichnofamily Iguanodontipodidae. This ichnofamily is typical for Cretaceous tracksites but the new material suggests that it might also be present in the Late Jurassic. The three morphotypes show a negative correlation between size and mesaxony, so the smaller tracks show the stronger mesaxony, and the larger ones weaker mesaxony. The Upper Jurassic ornithopod record from the Lusitanian Basin has yielded both small and medium-sized ornithopod remains, mainly iguanodontians such as dryosaurids and ankylopollexians, which are the main candidates to be the trackmakers.

Key words: Iguanodontipodidae, Dinehichnus, Anomoepus-like, Kimmeridgian, Tithonian, Europe.

Diego Castanera [diego.castanera@icp.cat; dcastanera@hotmail.es], Institut

Català de Paleontologia Miquel Crusafont, Universitat Autònoma de Barcelona, c/ Escola Industrial 23, 08201 Sabadell, Barcelona, Spain; Bayerische Staatssammlung für Paläontologie und Geologie and GeoBioCenter, Ludwig-Maximilians-Universität, Richard-Wagner-Str. 10, 80333 Múnich, Germany. Bruno C. Silva [laboratorio@alt-shn.org], Laboratório de Paleontologia e Paleoecologia, Sociedade de História Natural, Apartado 2564-909 Torres Vedras, Portugal; European Centre of Paleontology, Institute of Biology, Laboratory of 
Paleobiology, University of Opole, ul. Oleska 48, 45-052 Opole, Poland. Vanda F.

Santos [vafsantos@fc.ul.pt], Departamento de Geologia, Faculdade de Ciências

da Universidade de Lisboa, Edifício C6, Piso 3, Campo Grande, 1749-016

Lisboa, Portugal; Instituto Dom Luiz (IDL), Faculdade de Ciências da

Universidade de Lisboa, Campo Grande, Lisboa, Portugal. Elisabete Malafaia [emalafaia@gmail.com

], Instituto Dom Luiz (IDL), Faculdade de Ciências da Universidade de

Lisboa, Campo Grande, Lisboa, Portugal; Grupo de Biología Evolutiva.

Facultad de Ciencias. Universidad Nacional de Educación a Distancia.

C/ Senda del Rey, 9, 28040 Madrid, Spain. Matteo Belvedere [matteo.belvedere@ hotmail.com]; Institute for Studies in Landscape and Human Evolution, Faculty of Science and Technology, Bournemouth University, Talbot Campus, Fern Barrow, Poole, BH12 5BB, UK.

This is an open-access article distributed under the terms of the Creative Commons Attribution License (for details please see creativecommons.org), which permits unrestricted use, distribution, and reproduction in any medium, provided the original author and source are credited.

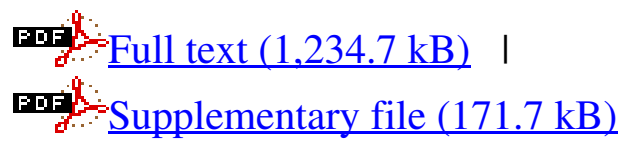

